\title{
Variações na Composição Isotópica da Precipitação e nas Águas Subterrâneas em Áreas de Recarga do Sistema Aquífero Guarani (SAG), na Porção Central do Estado de São Paulo
}

\author{
Sarah Maria Rodrigues dos Santos ${ }^{1}$; Ludmila Vianna Batista²; Vinícius dos Santos ${ }^{3}$; João Felipe \\ Gromboni $^{4}$; Bárbara Saeta Farinha ${ }^{5}$; Chang Hung Kiang ${ }^{6}$; Didier Gastmans ${ }^{7}$
}

\begin{abstract}
Resumo:
Isótopos estáveis vem sendo desde muito utilizados como traçadores do ciclo hidrológico devido as variações observadas na composição isotópica da água devido ao fracionamento isotópico. O presente estudo avaliou as variações na composição isotópica da precipitação e nas águas subterrâneas em área de afloramento do Sistema Aquífero Guarani, na cidade de Brotas (SP), buscando uma melhor compreensão nos mecanismos de recarga. O período de monitoramento foi caracterizado por precipitações abaixo da média, que se refletiram no rebaixamento dos níveis d'água, interrompido no verão 2014/2015. As variações medidas na composição isotópica da precipitação $\left(\sim 10 \%\right.$ o para $\delta^{18} \mathrm{O}$ e $80 \%$ para $\left.\delta^{2} \mathrm{H}\right)$ são muito maiores que aquelas das águas subterrâneas ( 1\%o para $\delta^{18} \mathrm{O}$ e 5 \%o para $\left.\delta^{2} \mathrm{H}\right)$. As águas subterrâneas apresentam razões isotópicas médias mais depletadas $\left(\delta^{18} \mathrm{O}=-7,25 \%\right.$ VSMOW e $\delta^{2} \mathrm{H}=-49,5 \%$ VSMOW) que as da precipitação, entretanto esses valores são semelhantes aos medidos na precipitação durante a estação chuvosa (entre novembro/2014 e maio/2015), quando se observa a recuperação dos níveis d'água.
\end{abstract}

\section{Abstract:}

Stable isotopes have been widely used as tracers of the hydrological cicle due to the observed variations in water isotopic composition associated to the isotopic fractionation. This study has evaluated variations in the precipitation and groundwater isotopic composition in Guarani Aquifer

\footnotetext{
${ }^{1}$ Graduação em Geologia - Univ. Estadual Paulista. Av. 24A, 1515 - Bela Vista - CEP: 13.500-900 - Rio Claro (SP). Fone: (19) 3526-9496. Email: sarah.mrds@hotmail.com

${ }^{2}$ Doutoranda em Geociências e Meio Ambiente - Univ. Estadual Paulista. Av. 24A, 1515 - Bela Vista - CEP: 13.500-900 - Rio Claro (SP). Fone: (19) 3526-9496. Email: ludvbatista@yahoo.com.br

${ }^{3}$ Mestrando em Geociências e Meio Ambiente - Univ. Estadual Paulista. Av. 24A, 1515 - Bela Vista - CEP: 13.500-900 - Rio Claro (SP). Fone: (19)

3526-9496. Email: vinicius.santos2130@yahoo.com.br

${ }^{4}$ Graduação em Geografia - Univ. Estadual Paulista. Av. 24A, 1515 - Bela Vista - CEP: 13.500-900 - Rio Claro (SP). Fone: (19) 3526-9496. Email: joaogromboni@gmail.com

${ }^{5}$ Graduação em Ecologia - Univ. Estadual Paulista. Av. 24A, 1515 - Bela Vista - CEP: 13.500-900 - Rio Claro (SP). Fone: (19) 3526-9496. Email: bsaetafarinha@gmail.com

${ }^{6}$ LEBAC - Univ. Estadual Paulista. Av. 24A, 1515 - Bela Vista - CEP: 13.500-900 - Rio Claro (SP). Fone: (19) 3526-9496. Email: chang@ @ rc.unesp.br

${ }^{7}$ Centro de Estudos Ambientais - - Univ. Estadual Paulista. Av. 24A, 1515 - Bela Vista - CEP: 13.500-900 - Rio Claro (SP). Fone: (19) 3526-9496.

Email: gastmans@rc.unesp.br
} 
System (GAS) outcrop area, located in Brotas (SP), seeking a better understanding on the recharge mechanisms. The monitoring period was characterized by rainfall under the historical average, which reflect in depletion of groundwater levels, interrupted during the summer season 2014/2015, when a recovery in groundwater levels were observed. Variations in isotopic composition of precipitation ( $10 \%$ or for $\delta^{18} \mathrm{O}$ and $80 \%$ for $\left.\delta^{2} \mathrm{H}\right)$ are larger than the observed in groundwater $\left(\sim 1 \%\right.$ for $\delta^{18} \mathrm{O}$ and 5 $\%$ for $\left.\delta^{2} \mathrm{H}\right)$. Groundwater presents more depleted mean isotopic ratios $\left(\delta^{18} \mathrm{O}=-7,25 \%\right.$ VSMOW and $\delta^{2} \mathrm{H}=-49,5 \%$ VSMOW) than the precipitation, however these values are similar to those measured in precipitation during the rainy season (from November/2014 to May/2015), when a recovery of groundwater levels was observed.

\section{Palavras Chave:}

Isótopos Estáveis, Precipitação, Recarga

\section{INTRODUÇÃO}

Os isótopos estáveis dos elementos constituintes da molécula da água $\left({ }^{1} \mathrm{H},{ }^{2} \mathrm{H},{ }^{16} \mathrm{O},{ }^{17} \mathrm{O}\right.$ e $\left.{ }^{18} \mathrm{O}\right)$ vem sendo desde muito utilizados como traçadores do ciclo hidrológico, uma vez que mudanças no estado físico da água resultam em alterações nas razões isotópicas, devido ao processo conhecido como fracionamento isotópico, que faz com que os distintos isótopos tenham comportamento diferenciados em função da temperatura, deslocando-se preferencialmente quando da mudança do estado da água (Mook, 2000).

Sua aplicação em estudos hidrológicos e climáticos iniciou-se a partir da instalação da Rede Global de Isótopos na Precipitação (Global Network of Isotopes in Precipitation - GNIP) no ano de 1958 pela Agência Internacional de Energia Atômica (International Atomic Energy Agency - IAEA) em cooperação com a Organização Meteorológica Mundial (World Meteorological Organization WMO). A GNIP tinha como objetivo inicial o monitoramento das concentrações de trítio produzido pelos testes nucleares na atmosfera, e posteriormente a composição isotópica de oxigênio e hidrogênio na precipitação. Atualmente com o advento da técnica da Espectrometria de Absorção Laser (Laser Absorption Espectroscopy), a capacidade de determinação de um número maior de amostras, faz com que novas fronteiras se abram para a utilização de isótopos estáveis em estudos hidrológicos e climáticos (Wassenar et al., 2014).

O armazenamento de um grande número de observações sobre a composição isotópica da precipitação na GNIP, possibilitou a constatação da existência de inúmeras relações entre a composição isotópica da precipitação e parâmetros climáticos, como a temperatura do ar ou a quantidade de precipitação, bem como a origem das massas de chuva, proporcionando dessa forma uma associação entre as condições climáticas e a precipitação (Dangsaard, 1964; Craig, 1961; Craig 
\& Gordon, 1965; Rozanski et al., 1992; Rozanski et al., 1993; Gat, 1996; Clark \& Fritz, 1997, dentre outros). Essas relações fazem da avaliação da composição isotópica da precipitação uma informação auxiliar na interpretação dos controles climáticos, especialmente devido a extensão limitada, tanto temporal quanto espacial, da rede de monitoramento de registros de parâmetros climáticos. A avaliação conjunta de registros preservados em arquivos paleoclimáticos, como espeleotemas e águas subterrâneas, pode ser utilizado como ferramentas auxiliares na interpretação de condições climáticas do Pleistoceno/Holoceno. (Aggarwal et al., 2012).

As variações observadas na composição isotópica da precipitação, relacionadas a variabilidade climática, influencia diretamente a composição isotópica das águas subterrâneas, apesar da atenuação observada no sinal isotópico quando da recarga, que faz com que as razões isotópicas observadas representem médias das precipitações anuais. Entretanto grandes variações em escalas milenares são facilmente reconhecidas ao longo de inúmeros aquíferos, como é o caso do Sistema Aquífero Guarani (SAG), onde águas isotopicamente depletadas, cerca de -3\% VSMOW em $\delta^{18} \mathrm{O}$, são encontradas em áreas próximas as áreas de recarga da unidade (Chang et al., 2012).

Nesse sentido, o presente trabalho tem por objetivo apresentar os resultados preliminares de programa de monitoramento temporal da composição isotópica da precipitação e de águas subterrâneas do Sistema Aquífero Guarani na cidade de Brotas (SP), realizado entre fevereiro de 2014 e dezembro de 2015.

\section{MATERIAIS E MÉTODOS}

O município de Brotas está localizado na porção central do Estado de São Paulo, inserida na bacia Hidrográfica do Rio Tietê-Jacaré (BH-TJ) - UGRHI-13 (Figura 1). Geologicamente o município encontra-se assentada predominantemente sobre os sedimentos clásticos arenosos das formações Botucatu e Pirambóia, constituintes do Sistema Aquífero Guarani e sobre as rochas ígneas basálticas da Formação Serra Geral, que constituem a sequencia mesozoica da Bacia Sedimentar do Paraná. A precipitação média anual é de $1510 \mathrm{~mm} / \mathrm{ano}$, distribuída ao longo do ano em duas estações: uma chuvosa, entre os meses de dezembro a fevereiro, concentrando cerca de $50 \%$ de toda a precipitação anual, e uma estação mais seca, que se estende de junho a agosto, quando chove aproximadamente 5\% do índice anual (SIGRH, 2005).

Para a coleta das amostras de precipitação foi utilizado um coletor especialmente desenhado para se evitar a evaporação, uma vez que a mesma altera a composição isotópica original da amostra coletada devido ao fracionamento ocorrido (Gröning et al, 2012) (Figura 1). As amostras de chuva foram coletadas mensalmente, em alíquotas de $50 \mathrm{~mL}$, filtradas com filtro seringa de acetato de celulose, abertura $0,45 \mu \mathrm{m}$ e armazenadas em frascos de vidro âmbar com dupla vedação, evitando- 
se grandes volumes de ar no interior do frasco, de modo a evitar a ocorrência de fracionamento por evaporação.

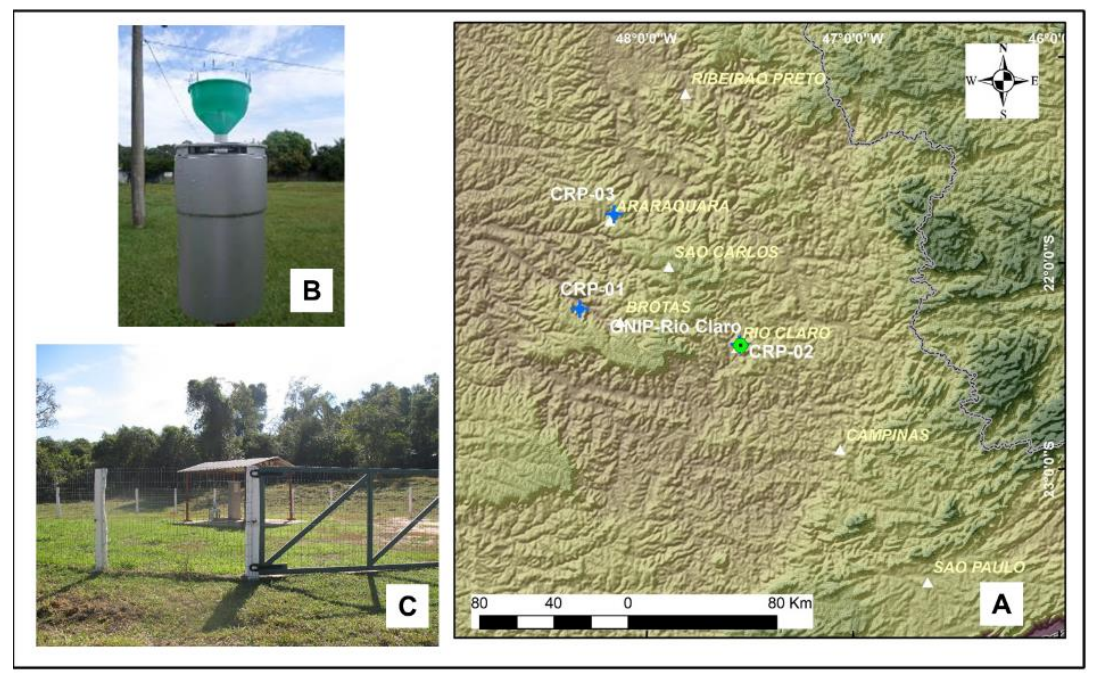

Figura 1. (A) Localização da área de estudo. (B) Coletor utilizado na amostragem da precipitação. (C) Poço monitorado

As amostras de água subterrânea foram coletadas mensalmente, nos primeiros dias do mês, em um poço tubular com 30 metros de profundidade, perfurado em arenitos da Formação Botucatu. Antes da coleta da amostra foi efetuada a medição dos níveis d'água e da condutividade elétrica, e o para se evitar a amostragem de água estagnada no interior do poço, o mesmo era bombeado por cerca de 20 minutos, garantindo que a amostra representasse a água contida no aquífero.

O conteúdo isotópico das amostras foi determinado por Espectrometria Laser de Cavidade Oca (Cavity Ring Laser Spectroscopy), no Laboratório de Hidrogeologia e Hidroquímica do Departamento de Geologia Aplicada do IGCE/UNESP, e os resultados expressos de acordo com a notação $\delta \%$ em relação ao VSMOW (Vienna Standard Mean Ocean Water), calculada de acordo com a equação 1 :

$$
\delta_{\text {amostra }}=\frac{R_{\text {amostra }}-R_{\text {padrão }}}{R_{\text {padrão }}} \times 1000
$$

Dados climáticos, como: temperatura, umidade, pressão atmosférica e precipitação, foram obtidos a partir de estação meteorológica automática modelo DRIA-0511, instalada no mesmo local da coleta das amostras de precipitação.

\section{RESULTADOS E DISCUSSÕES}

No período de monitoramento a precipitação observada foi de $980 \mathrm{~mm}$ durante o ano de 2014 e de 1050 mm no ano de 2015, ambos os anos bem abaixo da média observada na região. Os meses mais chuvosos foram fevereiro e dezembro/2014, com 198 e $166 \mathrm{~mm}$ respectivamente, e fevereiro e 
novembro/2015, com índices que alcançaram 179 e $205 \mathrm{~mm}$, respectivamente. Os meses mais secos foram junho de 2014 e 2015, quando não foi registrada precipitação.

Os baixos índices pluviométricos do período refletiram-se no comportamento dos níveis d'água subterrânea do poço monitorado, que apresentou profundidade variando entre $8,85 \mathrm{~m}$ até 9,69m, mostrando comportamento concordante com as taxas de precipitação. Observou-se no período uma tendência a rebaixamento contínuo dos níveis, com pequena recuperação entre os meses de janeiro e março/2015, efeitos da precipitação ocorrida a partir dos meses de novembro/2014 (Figura 2).

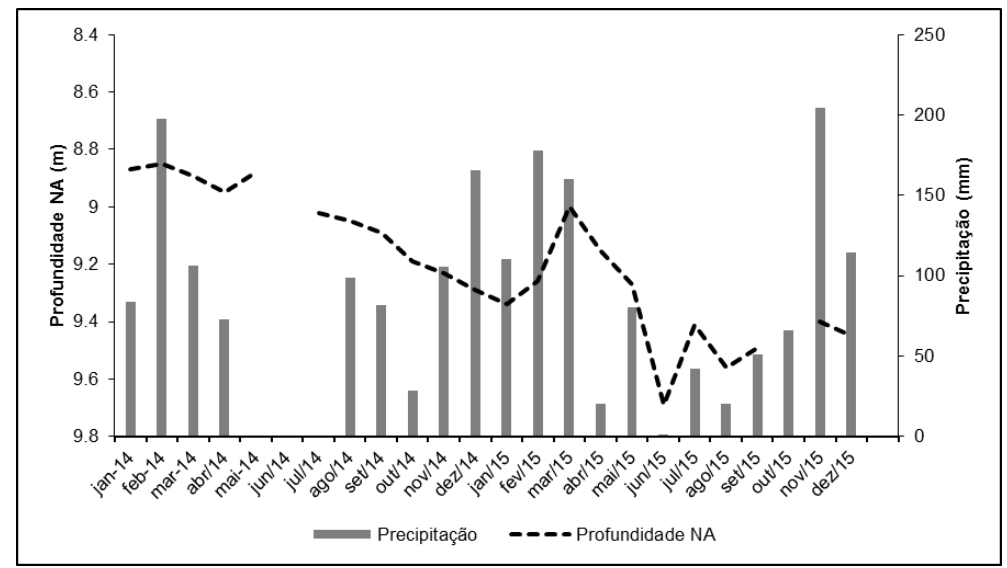

Figura 2. Variação da precipitação e da profundidade do NA no poço observado.

A composição isotópica da precipitação variou de $+0,02$ a -9,26\% VSMOW para $\delta^{18} \mathrm{O}$ e de $+17,3$ a $-63,3 \%$ VSMOW para $\delta^{2} \mathrm{H}$, com médias ponderadas de $-4,35 \%$ o VSMOW para $\delta^{18} \mathrm{O}$ e de 21,29 \% VSMOW para $\delta^{2} \mathrm{H}$. Observa-se ainda que os valores mais depletados estão associados ao período chuvoso novembro/2014 a abril/2015, que apresenta valores de $\delta^{18} \mathrm{O}$ aproximadamente 4\%o mais empobrecida que a precipitação do final do período chuvoso de 2013-2014 (Figura 3). Apesar dessas variações não foi observada boa correlação entre a quantidade de precipitação e os valores de $\delta^{18} \mathrm{O}\left(\mathrm{r}^{2}=0,07\right)$.

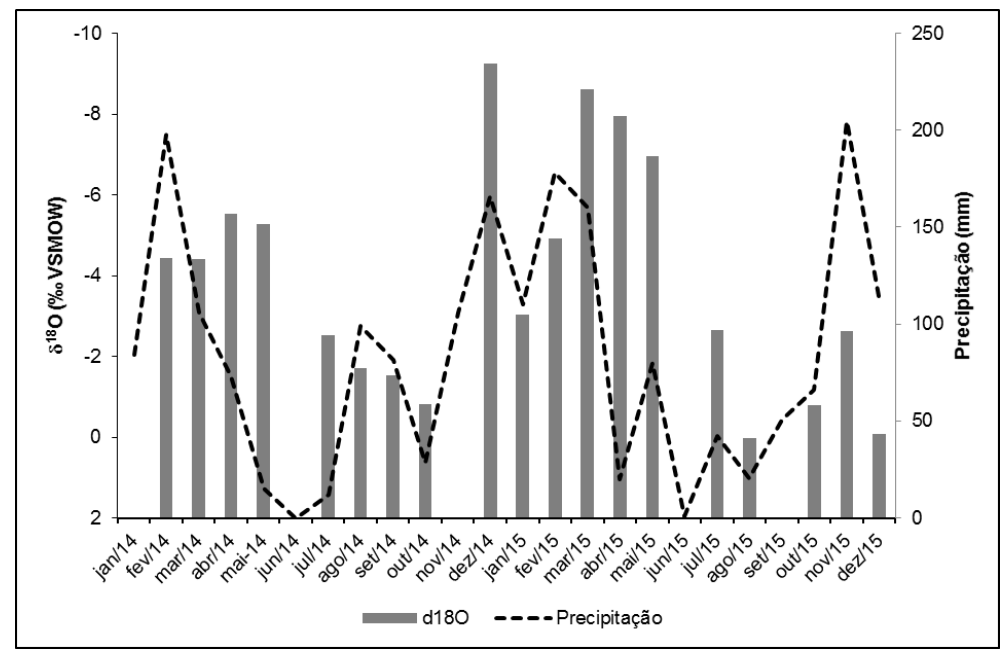

Figura 3. Variação da razão isotópica $\delta^{18} \mathrm{O}$ e da quantidade de precipitação 
A reta meteórica local $(\delta 2 \mathrm{H}=8,07 \delta 18 \mathrm{O}+14,33)$ apresenta inclinação aproximadamente igual a reta meteórica global, entretanto o excesso de deutério é um maior, indicando que a origem da precipitação possui fontes de vapor que passaram por processos de evaporação e recirculação em áreas continentais (Figura 4).



Figura 4. Reta meteórica local

A composição isotópica das águas subterrâneas variou de $-6,77$ a -7,76\%o VSMOW para $\delta^{18} \mathrm{O}$ e de $-44,7$ a $-49,5 \%$ VSMOW para $\delta^{2} \mathrm{H}$, com médias de $-7,25 \%$ VSMOW para $\delta^{18} \mathrm{O}$ e de $-49,5 \%$ o VSMOW para $\delta^{2} \mathrm{H}$ (Figuras 4 e 5). Essa pequena variação observada nas razões isotópicas das águas subterrâneas se explica pela atenuação do sinal isotópico durante o processo de recarga, quando ocorre a mistura de águas.

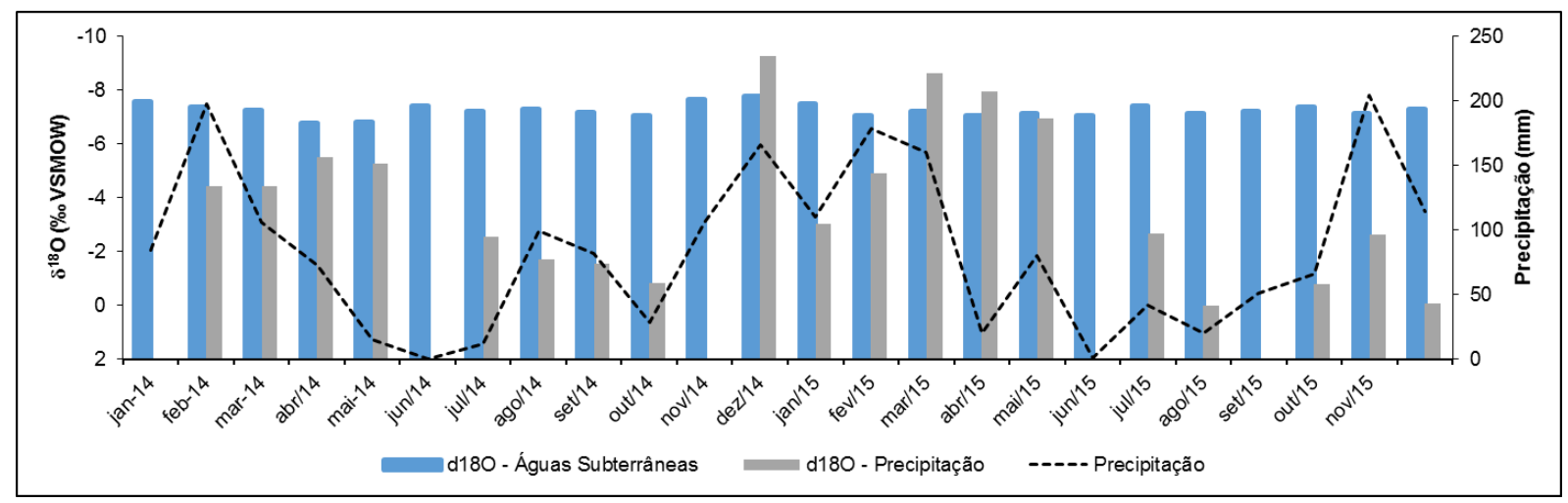

Figura 5. Variação temporal de $\delta^{18} \mathrm{O}$ nas águas subterrâneas e na precipitação

A composição isotópica das águas subterrâneas é aproximadamente a mesma da precipitação dos meses de novembro/2014 a maio/2015 (Figura 5), indicando que eventos de recarga ocorrem associados a maiores eventos de precipitação, como esperado, associado a recuperação dos níveis d'água observado no poço (Figura 2). 


\section{CONCLUSÕES}

A análise das variações na composição isotópica da precipitação e das águas subterrâneas, aliado ao monitoramento dos níveis d'água e dos índices pluviométricos, permitiu avaliar, de maneira preliminar as condições de recarga em área de afloramento do Sistema Aquífero Guarani, na cidade de Brotas (SP).

Os resultados do estudo indicam que as variações na composição isotópica da precipitação ( $10 \%$ para $\delta^{18} \mathrm{O}$ e 80 \% para $\left.\delta^{2} \mathrm{H}\right)$ são muito maiores que as observadas nas águas subterrâneas ( 1\%o para $\delta^{18} \mathrm{O}$ e $5 \%$ para $\left.\delta^{2} \mathrm{H}\right)$. A reta meteórica local apresenta inclinação próxima à da reta meteórica global, com excesso de deutério superior, indicando a existência de processos associados a origem do vapor distintos. Os valores mais depletados da precipitação foram observados no período chuvoso, durante o qual se observa a instalação da Zona de Convergência do Atlântico Sul, caracterizado pela ocorrência de chuvas convectivas. As razões isotópicas das águas subterrâneas são semelhantes as observadas nessas precipitações, e são coincidentes com o período em que foi observada a recuperação dos níveis d'água no aquífero.

\section{AGRADECIMENTOS}

Os autores gostariam de agradecer a Agência Internacional de Energia Atômica (IAEA) (CRP19747 "Stable isotopes in precipitation and paleoclimatic archives in tropical areas to improve regional hydrological and climatic impact models") e a Fundação de Amparo à Pesquisa do Estado de São Paulo - FAPESP (Processo 2015/15749-2) pela concessão de auxílio financeiro para a realização do projeto, ao CNPq pela concessão de bolsa PIBIC (Processo 33307) ao primeiro autor, a CAPES pela concessão de bolsa ao segundo autor, a FAPESP pela concessão de bolsa ao terceiro e quarto autores (Processos 2013/06704-0 e 2016/01195-8)

\section{REFERÊNCIAS BIBLIOGRÁFICAS}

Aggarwal, P.K., Alduchov, O.A., Froehlich, K.O., Araguas-Araguas, L.J., Sturchio, N.C., Kurita, N. Stable isotopes in global precipitation: a unified interpretation based on atmospheric moisture residence time. Geophysical Research Letters, 39, L11705, doi:10.1029/2012GL051937. 2012.

Chang, H.K.; Aravena, R., Gastmans, D., Hirata, R., Manzano, M., Vives, L., Rodrigues, L., Aggarwal, P, Araguas, L. Role of isotopes in the development of a general hydrogeological conceptual model of the Guarani Aquifer System (GAS) In: Isotopes in Hydrology, Marine Ecosystems and Climate Change Studies.1 ed. Viena : International Atomic Energy Agency, 2013, v.2, p. 281-290. 2013.

Craig, H. Isotopic variations in meteoric waters. Science, 133: 1702-1703. 1961. 
Craig, H., Gordon, L. I. Deuterium and oxygen 18 variations in the ocean and marine atmosphere. In proc. Stable Isotopes in Oceanographic Studies and Paleotemperatures, 1965, Spoleto, Italy. edited by E. Tongiogi, pp 9-130, V. Lishi e F., Pisa. 1965.

Dansgaard, W. Stable isotopes in precipitation. Tellus, 16, 436-468, doi:10.1111/j.21533490.1964.tb00181.x, 1964.

Fritz, P., Clark, I. D. Environmental Isotopes in Hydrogeology, Boca Raton, Florida: Lewis Publishers, 1997. 325p. 1997.

Gat, J. R.: Oxygen and hydrogen isotopes in the hydrologic cycle, Annual Review of Earth and Planetary Science, 24, 225-262, doi:10.1146/annurev.earth.24.1.225, 1996.

Gröning, M.; Lutz, H.O.; Roller-Lutz, Z.; Kralik, M.; Gourcy, L.; Pöltenstein, L. A simple rain collector preventing water re-evaporation dedicated for $\delta^{18} \mathrm{O}$ and $\delta^{2} \mathrm{H}$ analysis of cumulative precipitation samples. Journal of Hydrology, 448-449: 195-200. 2012.

Mook, W.G (ed.) Environmental Isotopes in Hydrological Cycle - Principles and Aplications. IHPV Technical Documents in Hydrology, no 39, UNESCO-IAEA. 596p. 2000.

Rozanski, K., Sonntag, C., and Münnich, K.O. Factors controlling isotopic composition of European precipitation. Tellus, 34: 142-150. doi: 10.1111/j.2153-3490.1982.tb01801.x. 1982.

Rozanski, K., Araguas-Araguas, L.J., and Gonantini, R. Isotopic patterns in modern global precipitation. In Climate Change in Continental Isotopic Records, Swart PK, Lohmann KC, McKenzie J Savin S (eds) Geophysical Monograph No 67. American Geophysical Union. Washington: 1-36. 1993.

Wassenaar, L.I.; Coplen, T.B.; Aggarwal, P.K. Approaches for achieving long-term accuracy and precision of $\delta^{18} \mathrm{O}$ and $\delta^{2} \mathrm{H}$ for waters analyzed using Laser Absorption Spectrometers. Enviromental Science \& Tecnology. 48:1123-1131, doi: dx.doi.org/10.1021/es403354n. 2014 